\title{
How might epigenetic dysregulation in early embryonic life contribute to autism spectrum disorder?
}

\author{
"The potential role for epigenetic dysregulation contributing to the pathogenesis of \\ autism spectrum disorder is a current major focus of interest."
}

Keywords: autism $\bullet$ DNA methylation $\bullet$ embryo $\bullet$ epigenetic

The potential for nongenetic susceptibility to mediate part of the risk of autism spectrum disorder (ASD) has prompted a number of studies to date, all showing evidence for epigenetic differences characterizing the individuals with ASD. The modest differences in DNA methylation observed have indicated an underlying cellular mosaicism for epigenetic dysregulation. The studies to date have not comprehensively addressed potential confounding issues like cell subcomposition differences, transcriptional and DNA sequence variability. Additionally, it is possible that mutations in proteincoding genes encoding transcriptional and chromatin regulatory proteins lead to the epigenetic changes in a subset of individuals with ASD. More definitive studies are now needed to allow higher confidence insights into epigenetic events occurring in early embryogenesis in individuals with ASD.

Not all problems with genes have to do with their DNA sequence. If a gene with a completely normal DNA sequence capable of encoding a functional protein cannot be switched on appropriately, the effect for the cell is likely to be the same as a loss of function mutation of that gene. Problems of this type that remain heritable through cell divisions have been attributed to the subset of transcriptional regulatory mechanisms that are described as 'epigenetic.' DNA methylation has transcriptional regulatory properties and biochemical properties that are genuinely epigenetic [1], whereas other transcriptional regulatory mechanisms have not been proven to have the same heritability at the molecular level, although there is some evidence for heritability mechanisms of some histone post-translational modifications [2]. Genome-wide studies of these potentially epigenetic regulatory processes define the 'epigenome,' and represent a first means of seeking a role for abnormal regulation of transcription in phenotypic and disease studies.

The potential role for epigenetic dysregulation contributing to the pathogenesis of autism spectrum disorder (ASD) is a current major focus of interest. This interest is in part driven by the desire to understand why an individual developed ASD in the first place, and in part by the recognition that the inherently malleable epigenome may be a target for intervention to reverse any deleterious molecular events, potentially allowing amelioration of the ASD phenotype. Furthermore, as we try to understand how the broad and vague category of 'environmental' agents influence the risk of susceptibility to ASD [3], the epigenome is an attractive candidate for study as a potential mediator of these influences.

There have now been several studies of epigenetic dysregulation in ASD. An early study looked at trimethylation of the lysine at position 4 of the histone $\mathrm{H} 3$ protein (H3K4me3) in neuronal nuclei isolated from prefrontal cortex from post mortem brains of 16 subjects with ASD and 16 controls. Sequencing of the DNA enriched by chromatin immunoprecipitation (ChIP-seq) showed a broadening of the $\mathrm{H} 3 \mathrm{~K} 4 \mathrm{me} 3$ peaks at gene promoters in some of the ASD subjects, providing a suggestion that there could be abnormal regulation

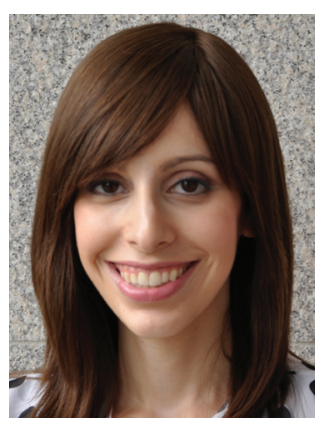

Esther R Berko

Department of Genetics,

Albert Einstein College of Medicine, Bronx, NY 10461, USA and

Department of Pediatrics, Columbia University College of Physicians \& Surgeons, New York, NY 10032, USA

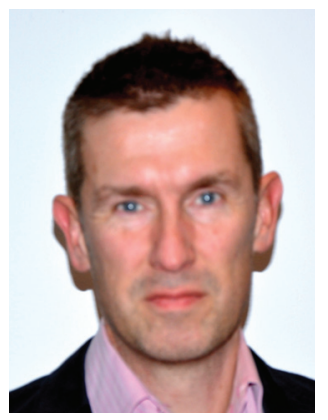

John M Greally

Author for correspondence: Department of Genetics, Albert Einstein College of Medicine, Bronx, NY 10461, USA Tel.: +1 7186781234 john.greally@einstein.yu.edu
Future
Medicine fss 
of transcription in brains of individuals with ASD [4]. DNA methylation studies of peripheral blood leukocytes [5], brain [6,7] and ectodermally-derived buccal epithelial cells [8] from individuals with ASD and controls have all subsequently been performed. While each study had its own distinctive technical and analytical approaches, they all showed subsets of loci with distinctive changes in DNA methylation associated with ASD, with two of the studies converging upon the same OR2L13 gene as a target for epigenetic changes $[5,8]$. The other consistent finding was that the degree of change in DNA methylation observed distinguishing the ASD from control individuals was only of modest magnitude. This is an interesting finding, as it implies that only a subset of cells tested is changing DNA methylation in the individuals with ASD [8], a mosaicism for cellular dysfunction that could provide an interesting insight into disease pathogenesis.

"This interest is in part driven by the desire to understand why an individual developed autism spectrum disorder in the first place, and in part by the recognition that the inherently malleable epigenome may be a target for intervention to reverse any deleterious molecular events, potentially allowing amelioration of the autism spectrum disorder phenotype."

The question then arises about how we should be interpreting these findings. If there are histone posttranslational or DNA methylation differences occurring in subsets of cells in the body of an individual with ASD, does this give us an insight into disease mechanism? A first way of addressing this question is to ask what reason might exist for epigenetic dysregulation in individuals with ASD. In our previous work, we focused on individuals with ASD whose mothers were at least 35 years of age at the time of their delivery [8], one possibility being that the increased risk associated with older mothers [9] could reflect an ageassociated drift of the oocyte epigenome. Other environmental influences have been invoked as potential reasons for ASD susceptibility [3], although the mechanistic links to epigenetic regulators are often not well supported at the molecular level at present, and are not easily invoked as explanations for the studies published to date.

An intriguing possibility is that some or all of the changes in DNA methylation and histone post-translational modifications are secondary to mutations in genes regulating chromatin function. The frequency of mutations in this group of transcriptional regulatory genes has been notable [10,11], with a recent study of 3871 individuals of ASD revealing a substantial proportion to have mutations in genes encoding proteins involved in transcriptional and chromatin regulation [12]. While the effects of mutations of these genes upon $\mathrm{H} 3 \mathrm{~K} 4 \mathrm{me} 3$ or DNA methylation are not well understood, it is certainly a possibility that epigenomic abnormalities are reflective of mutations in genes regulating chromatin and transcription, at least in some individuals.

The other possibility is that the epigenomic dysregulation observed is indicative of other influences. We now appreciate that DNA methylation can be influenced by cell subcomposition variability [13,14], transcriptional variability [15-17] and by DNA sequence variants, which have been estimated to account for anything from 22 to $80 \%$ of DNA methylation variability between individuals [18-20]. The previous epigenome-wide studies of ASD have included measures that either accounted for cell subcomposition analytically [6] or used an homogeneous cell type [8], and also included an adjustment for ancestry [8] to help reduce the effect of genetic polymorphism. As studies continue to improve, taking account of these known and other potential sources of variability, we can expect higher confidence findings as we dissect the role of the epigenome in ASD.

So how do we do better in performing the studies that allow us to ask whether epigenetic dysregulation early in life has a role in ASD? The first answer has to do with principles of experimental design. If we know that cell subcomposition is a potential source of variability, we need to address this, the rigorous approach being to select homogeneous cells for analysis. If transcription and DNA sequence polymorphism are major potential influences upon DNA methylation, we need to perform transcriptional and sequencing studies concurrently upon the same cells, and power the studies adequately to allow high confidence findings to emerge. If the increasingly affordable option of whole genome sequencing is performed, we can add in the question whether mutations of proteins affecting transcriptional and chromatin regulators account for a subset of individuals with ASD in whom we observe epigenetic dysregulation. Ideally, we will have captured as much information from subjects and parents about histories of environmental exposures, allowing an exploration of possible influences upon the epigenomes of the individuals studied. We need to be open to the idea that the effects observed may reflect events in mosaic subpopulations of cells, not just epigenetic but also DNA sequence mutational events. The rich datasets generated by these more comprehensive approaches will present analytical challenges in dissecting out the relative contributions of the genome, 
transcriptome and epigenome, but should allow us to gain higher confidence insights into the intriguing possibility that the epigenome mediates part of the risk of ASD, and potentially even some information about the reasons why the epigenome is altered in the first place to create this risk.

\section{Conclusion}

Increasingly stringent studies of the epigenome have shown consistent evidence for distinctive patterns in individuals with ASD. At present, we do not know how to interpret these differences. The simple hypothesis that the epigenome is directly affected by an environmental influence and independently affects transcriptional regulation is complicated by our ongoing insights into other influences upon the epigenome, including cell subtype heterogeneity, and transcriptional and genetic sequence variability. We now also appreciate that the mutational events in protein-coding genes in ASD are potentially related to the epigenetic events observed in these individuals. Possibly, the most valuable insight from epigenomic studies to date is that they clearly implicate mosaic events in ASD, which could be relevant mechanistically for DNA sequence mutational studies. There is a clear need for more comprehensive studies that can dissect apart the numerous interacting mechanisms

\section{References}

1 Sharif J, Muto M, Takebayashi S et al. The SRA protein Np95 mediates epigenetic inheritance by recruiting Dnmt1 to methylated DNA. Nature 450(7171), 908-912 (2007).

2 Hansen KH, Bracken AP, Pasini D et al. A model for transmission of the H3K27me3 epigenetic mark. Nat. Cell Biol. 10(11), 1291-1300 (2008).

3 Grabrucker AM. Environmental factors in autism. Front. Psychiatry 3, 118 (2012).

4 Shulha HP, Cheung I, Whittle C et al. Epigenetic signatures of autism: trimethylated $\mathrm{H} 3 \mathrm{~K} 4$ landscapes in prefrontal neurons. Arch. Gen. Psychiatry 69(3), 314-324 (2012).

5 Wong CC, Meaburn EL, Ronald A et al. Methylomic analysis of monozygotic twins discordant for autism spectrum disorder and related behavioural traits. Mol. Psychiatry 19(4), 495-503 (2014).

6 Ladd-Acosta C, Hansen KD, Briem E, Fallin MD, Kaufmann WE, Feinberg AP. Common DNA methylation alterations in multiple brain regions in autism. Mol. Psychiatry 19(8), 862-871 (2014).

7 Nardone S, Sams DS, Reuveni E et al. DNA methylation analysis of the autistic brain reveals multiple dysregulated biological pathways. Transl. Psychiatry 4, e433 (2014).

8 Berko ER, Suzuki M, Beren F et al. Mosaic epigenetic dysregulation of ectodermal cells in autism spectrum disorder. PLoS Genet. 10(5), e1004402 (2014). and allow clear insights into the relative role of the epigenome on its own or in combination with other mediators of disease.

\section{Future perspective}

The next step for epigenome-wide association studies is to move into a new study design that adds DNA sequencing and transcriptional studies, what could be referred to as a functional genome-wide association study approach. Ideally, these would be performed upon single cell types mediating the phenotype, or a reasonable surrogate, in sufficiently large cohorts to allow confidence in findings. The greater the clinical and exposure information available from the families studied, the more potential for insights into influences upon the epigenome and outcomes of epigenetic dysregulation.

\section{Financial \& competing interests disclosure}

The authors have no relevant affiliations or financial involvement with any organization or entity with a financial interest in or financial conflict with the subject matter or materials discussed in the manuscript. This includes employment, consultancies, honoraria, stock ownership or options, expert testimony, grants or patents received or pending, or royalties.

No writing assistance was utilized in the production of this manuscript.

9 Sandin S, Hultman CM, Kolevzon A, Gross R, Maccabe JH, Reichenberg A. Advancing maternal age is associated with increasing risk for autism: a review and meta-analysis. J. Am. Acad. Child Adolesc. Psychiatry 51(5), 477-486 (2012).

10 Ben-David E, Shifman S. Combined analysis of exome sequencing points toward a major role for transcription regulation during brain development in autism. Mol. Psychiatry 18(10), 1054-1056 (2012).

11 Lasalle JM. Autism genes keep turning up chromatin. $O A$ Autism 1(2), 14 (2013).

12 De Rubeis S, He X, Goldberg AP et al. Synaptic, transcriptional and chromatin genes disrupted in autism. Nature 515(7526), 209-215 (2014).

13 Houseman EA, Accomando WP, Koestler DC et al. DNA methylation arrays as surrogate measures of cell mixture distribution. BMC Bioinformatics 13, 86 (2012).

14 Jaffe AE, Irizarry RA. Accounting for cellular heterogeneity is critical in epigenome-wide association studies. Genome Biol. 15(2), R31 (2014).

15 Zilberman D, Gehring M, Tran RK, Ballinger T, Henikoff S. Genome-wide analysis of Arabidopsis thaliana DNA methylation uncovers an interdependence between methylation and transcription. Nat. Genet. 39(1), 61-69 (2007).

16 Ball MP, Li JB, Gao Y et al. Targeted and genome-scale strategies reveal gene-body methylation signatures in human cells. Nat. Biotechnol. 27(4), 361-368 (2009). 
17 Suzuki M, Oda M, Ramos MP et al. Late-replicating heterochromatin is characterized by decreased cytosine methylation in the human genome. Genome Res. 21(11), 1833-1840 (2011)

18 Bell JT, Pai AA, Pickrell JK et al. DNA methylation patterns associate with genetic and gene expression variation in HapMap cell lines. Genome Biol. 12(1), R10 (2011).

19 Gertz J, Varley KE, Reddy TE et al. Analysis of DNA methylation in a three-generation family reveals widespread genetic influence on epigenetic regulation. PLoS Genet. 7(8), e1002228 (2011).

20 Grundberg E, Meduri E, Sandling JK et al. Global analysis of DNA methylation variation in adipose tissue from twins reveals links to disease-associated variants in distal regulatory elements. Am. J. Hum. Genet. 93(5), 876-890 (2013). 